\title{
Comprehension of conventional gestures in typical children, children with autism spectrum disorders and children with language disorders
}

Compréhension des gestes conventionnels chez les enfants typiques, les enfants avec troubles du spectre autistique et les enfants ayant des troubles du langage oral

\author{
Annabelle Perrault ${ }^{1,2}$, Laurence Chaby ${ }^{2,3}$, Fabienne Bigouret ${ }^{1}$, Alice Oppetit ${ }^{1}$, David Cohen ${ }^{1,2}$, Monique \\ Plaza $^{2}$, Jean Xavier ${ }^{1,2}$
}

1Département de Psychiatrie de l'Enfant et l'Adolescent, APHP, Groupe Hospitalier Pitié-Salpêtrière, Paris, France; 2Institut des Systèmes Intelligents et Robotique, ISIR, CNRS UMR 7222, Paris, France;

3Université Paris Descartes, Sorbonne Paris Cité, Institut de Psychologie, Boulogne-Billancourt, France.

Corresponding author:

Dr. Alice Oppetit

Département de Psychiatrie de l'Enfant et l'Adolescent

APHP, Groupe Hospitalier Pitié-Salpêtrière

47-83 Bd de l'hôpital, Paris 75013 - France

Phone: +33 (0)1 42162342

Email : alice.oppetit@aphp.fr 


\section{RÉSUMÉ (français)}

Contexte: Les gestes conventionnels contribuent à la communication et sont un précurseur du langage verbal. Le but de notre étude était: (a) d'analyser le développement de la compréhension des gestes conventionnels chez les enfants typiques (b) d'explorer cliniquement comment les enfants atteints de troubles du spectre autistique et de troubles du langage oral comprennent les gestes conventionnels.

Méthodes: La compréhension des gestes conventionnels a été évaluée au moyen d'un test vidéo. Le protocole consistait en quinze gestes arbitraires et quinze gestes iconiques. Nous avons recruté 262 enfants typiques âgés de 3 à 10 ans et 55 enfants atteints de troubles de la communication âgés de 4 à 8 ans: 36 enfants atteints de troubles du spectre autisitique et 19 atteints de troubles du langage oral.

Résultats: La compréhension des gestes conventionnels augmente au cours du développement chez les enfants typiques. L'amélioration de la compréhension des gestes iconiques survient plus tôt que celle des gestes arbitraires. Les enfants atteints de troubles du spectre autistique ont des scores de compréhension des gestes conventionnels inférieurs à ceux des enfants typiques et des enfants ayant des troubles du langage oral. Pour chaque geste pris séparément, les enfants atteints de troubles du langage ont de meilleurs résultats que les enfants autistes mais aussi que les enfants typiques. En outre, l'analyse de la compréhension des gestes à la fois arbitraire et iconique montre que les enfants avec trouble du langage oral ont des meilleurs scores que les enfants typiques ou avec troubles du spectre autistique.

Conclusions: Nos résultats suggèrent: (i) une dissociation entre la compréhension du langage oral et la compréhension gestuelle chez les enfants atteints de trouble du langage (ii) un déficit à la fois du langage oral et de la compréhension gestuelle chez les enfants atteints de troubles du spectre autistique.

Mots-clés: Trouble du spectre autistique, troubles de la communication, troubles du langage, gestes conventionnels, gestes iconiques, gestes arbitraires 


\section{ABSTRACT (english)}

Background : Conventional gestures (CG) contribute to communication and are a precursor of verbal language. The aim of our study was: (a) to analyse the developmental course of CG comprehension in typical children; (b) from a clinical perspective, to document how children with autism spectrum disorders (ASD) and language disorder (LD) can understand CG.

Methods : Comprehension of CG was evaluated through a video-based testing. The protocol consisted of fifteen arbitrary gestures and fifteen iconic gestures. We recruited 262 typical children aged from 3 to 10 years old and 55 children with communication impairments aged from 4 to 8 years old: 36 children with ASD and 19 with LD.

Results : CG comprehension increased during the early development of typical children and the improvement of iconic gestures comprehension occurred earlier than that of arbitrary ones. Children with ASD received lower CG scores than typical children and LD children. For each gesture taken separately, children with LD performed better than not only ASD children but typical children as well. Furthermore, when we performed the analysis with both arbitrary and iconic gesture, children with LD outperformed both children with ASD and TD children.

Conclusions: Our results suggest a dissociation between oral language comprehension and language gesture comprehension in children with LD and a deficit in both oral language and language gesture comprehension in children with ASD.

Keywords: Autism spectrum disorder, communication impairments, language disorders, conventional gestures development, iconic gestures, arbitrary gestures 


\section{INTRODUCTION}

To efficiently communicate and establish social interaction with others from an early age, individuals must be able to interpret both verbal and non-verbal messages [1,2]. Gestures constitute a universal and primary feature of human communication and a precursor to verbal language development [3]. Co-verbal gestures are defined as the arm and hand movements that synchronise with speech [4]. They serve the purpose of supplementing language content, regulating speech flow, maintaining the attention between a speaker and listener during a conversation, shifting a conversational topic, facilitating the continuation of a topic, and emphasizing a particular topic or content [5]. No categorisation of co-verbal gestures achieve unanimity between researchers. In this paper, we focus on conventional gestures (CG) [6], one of the 5 classes of co-verbal gestures according to the taxonomy of Ekman and Friesen [7]. CG gestures are intentional, communicative acts which have a direct verbal translation usually consisting of a word or a phrase and which can easily be understood without speech by a certain culture or social group.

\section{Conventional gestures during development}

Previous research has shown that gesture both precedes and is tightly related to changes in early language development [8,9]. Gesture comprehension emerges early (Morford \& Goldin-Meadow, 1992) and its developmental trajectory is tightly linked with that of gesture production (Dimitrova, Özçalışkan, Adamson, 2017). However, research on gesture comprehension remains relatively scarce compared to the large literature documenting developmental changes in gesture production. Young children typically begin to gesture between 8 and 12 months [10]. They first use deictic gestures (i.e. pointing gestures), whose meaning is given entirely by context and not by their form. Shortly after, between ages two to three, children produce and comprehend CG (Hodges, Özçalışkan \& Williamson, 2017).

CG may be coded in an arbitrary (e.g., moving the hand to say 'goodbye') or iconic (e.g.a hand holding a glass to express drinking) way [11]. Arbitrary means that the actual form of the symbol does not reflect the form of the thing or activity it symbolizes. On the other hand, iconic gestures show a close relation between the form or embodiment of the gesture and the meaning of its verbalisation. This type of gesture is produced when people move their arms and hands to produce a dynamic visual representation of the objects or events (action, size, motion, or shape) they wish to communicate [1,4]. Iconic gestures play an important role in precipitating early 
word learning [12]. Communication skills and the expression of conventional gestures significantly improve between the ages of three and four years [13], with children acquiring a growing number of gestures that can be used while speaking. From the age of 5, they possess a plateauing repertoire of approximately one hundred gestures knowing that several gestures will disappear and will be replaced by lexical productions [14]. When such lexicon is acquired, the remaining conventional gestures still perform several functions: they can replace, clarify or nuance language [15]).

According to Piaget's theory of symbolic development [16], one could suppose that iconic gestures are acquired earlier than arbitrary gestures because of their resemblance between the symbol and its referent (Hodges, Özçalışkan \& Williamson, 2017). In their study, Dimitrova et al. (Dimitrova, Özçalışkan, Adamson, 2017) found that, for 2- to 4-year-old children, comprehension of iconic gesture was better than that of arbitrary gestures. However, several studies indicate that children are good at acquiring both iconic and arbitrary gestures early in development, displaying no clear advantage for iconic gestures (Thompson, Vinson, Woll \& Vigliocco, 2012).

Namy, Campbell and Tomasello [17] conducted a study that examined mapping of iconic vs. arbitrary gestures in children aged 18 months, 26 months, and 4 years. They concluded that all ages were successfully able to map iconic gestures. However, only 18-month-olds and 4-year-olds, but not 26-month-olds, were able to map arbitrary gestures, revealing a U-shaped developmental function. The re-emergence of arbitrary gestures at 4 years is driven by a wider range of symbolic experiences and enhanced sensitivity to others' communicative intent.

Because CG contribute to communication and is a precursor of verbal language, their study and evaluation in children presenting neuro-developmental disorders including communication difficulties is crucial. According to DSM-5, communication disorders include several diagnosis among which language disorder (LD) and autism spectrum disorder. The former can affect both expressive and receptive language and is defined as a relatively 'pure' language impairment. ASD is characterised by severe deficits and pervasive impairment in several areas of development such as reciprocal social interactions, communication skills and stereotyped behaviors, interests and activities.

Pragmatics are defined as the use of language, prosody, and gesture for social communication. Deficits in pragmatics are considered a hallmark symptom of ASD and were once thought to distinguish ASD from LD. However, children with LD are a heterogeneous group in terms of linguistic abilities. Some children with LD 
may also have pragmatic difficulties $[18,19]$. What about the acquisition of conventional gestures in these two groups ASD and LD?

\section{Conventional gestures in Children with Language Disorders}

Children with LD should theoretically be able to use gesture as a compensatory mechanism providing an alternative mean to convey words or extend utterances [20]. Within the literature, there is some debate as to how and when children with LD use gesture during spontaneous communication. Gesture is a complex skill that requires the combination of social, cognitive and motor skills. Impairments concerning these skills are often comorbid with LD [21]. Studies suggest that, compared to typically developing (TD) children, children with LD exhibit poorer performance on gross and fine motor tasks and have difficulty producing hand sequences [22]. Some studies have found that children with LD produce significantly more gestures than their TD peers [23]. On the other hand, others argue that children with LD do not gesture more frequently than TD children [24], do not use gesture as a means of nonverbal communication, and, consequently, exhibit social difficulties [25]. Furthermore, some researchers have suggested that gesture and speech form an integrated communication system [4]. This theory would suggest that, similar to adults with aphasia for whom speech and gesture are impaired in parallel [4], children with language impairments may also display difficulties with gestural communication. Botting (Botting, Riches, Gaynor \& Morgan, 2010) explored gesture production and comprehension abilities in relation to LD in school-aged children (4.3-7.4 years old). They found that the gesture production and motor control scores of the LD group did not differ significantly from a group of agematched TD controls. However, differences were found on a comprehension task combining speech and gesture. The multimodal task consisted of a spoken sentence whose last word had been replaced with a gesture. Children were asked to identify the missing word from a choice of four pictures (including a semantic distractor, gesture distractor, and unrelated distractor). TD children achieved higher accuracy scores than peers with LD. In addition, when children failed to integrate gestural and spoken information in an utterance, the TD group was more likely to rely on the spoken cues and therefore was more likely to select the semantic distractor. In contrast, the children with LD were more likely to select the gesture distractor, suggesting that these children had failed to extract the intended gesture meaning. Finally, there were no gender differences in the gesture scores in either LD or TD group. Replicating this last study, Wray et al. [26] found support for the hypothesis of gesture and speech forming an integrated system: children with LD scored significantly lower than TD children on gesture- 
production and on gesture-comprehension tasks [27]. They concluded that LD children failed either to extract the movement meaning or to integrate the gesture meaning with semantic context.

\section{Conventional Gestures in Children with Autism Spectrum Disorders}

Children with ASD produce less proto-declarative pointing and less communicative gestures than TD children, as described in studies based on familial home movies focusing on early signs of autism [28]. However, they are able to formulate demands in protest by using proto-imperative gestures and unfamiliar gestures such as pushing an adult's hand towards a desired object or using the other as a tool [29].

Clinicians have long reported abnormalities in both verbal and nonverbal forms of communication in ASD, including the 'clumsy' and 'inappropriate' nature of gestures described by Asperger in 1944 [30]. The presence of communicative gesture deficits in ASD is widely asserted in the clinical literature [31]. The impairments in ASD's gestures include (i) atypical gesture and/or developmental delay of the gesture [32,33] (ii) poor integration with speech, (iii) reduced frequency [34] and (iv) poor variety [35]. Impairments in gesture are codified on gold-standard ASD diagnostic measures such as the ADOS [36], the Autism Diagnostic Interview [37], and the M-CHAT [38]. In these questionnaires, the absence or infrequency of gesture is rated as symptomatic. Scoring criteria for these diagnostic measures suggest that individuals with ASD use less gestures than their typically developing (TD) peers. Many studies using only parent report [33,39] emphasized on pointing and showing gestures [40,41]. Moreover, compared to gesture production, gesture comprehension in children with ASD remains an understudied research domain (Dimitrova, Özçalışkan, Adamson, 2017). Given the literature regarding conventional gestures of both LD and ASD, we believe that conventional gesture impairments could constitute a significant clinical dimension to better differentiate LD and ASD communication impairments. Early screening could also be useful in distinguishing the developmental trajectory of these two neurodevelopmental disorders as well as exploring their possible overlap that could aid clinicians in early diagnosis and tailored remediation.

\section{Aims of the Study}

To date, little is known about the development of CG comprehension especially in children with LD and ASD, and there is no established means of assessing it. Thus, the first aim of the present study was to analyse the developmental course of CG comprehension (from age 3 to 10 years) using an experimental protocol specifically designed for this purpose. The second aim was to analyse, from a clinical perspective, how children with 
developmental communication disorders can understand conventional gestures, insomuch as this ability can compensate for language weakness or absence. We formulated the following hypotheses: (i) among TD children, the comprehension of conventional gestures would quantitatively and qualitatively increase during development (ii) iconic conventional gestures would be acquired before arbitrary gestures in TD, ASD, and LD children (iii) among children with ASD, comprehension of iconic and arbitrary conventional gestures would be lower to that of TD children (iv) children with LD would correspond to an intermediate profile between ASD and typical children, showing better performance than children with ASD but worse or comparable performance compared to typical children of similar developmental age. 


\section{METHODS}

\section{Participants}

To perform a developmental analysis of the experimental procedure, we recruited 262 typical children (119 girls and 143 boys) from 3 to 10 years $(3$ y.o, $n=11 ; 4$ y.o. $n=62 ; 5$ y.o. $n=55 ; 6$ y.o. $n=39 ; 7$ y.o. $n=52 ; 8-10$ y.o. $n=37)$.

The typical children were enrolled in the ordinary educational system and their educational levels corresponded to their age. We also recruited 55 children, from 4 to 8 years old, with communication impairments: 36 with $\operatorname{ASD}$ (mean $=6 \pm 1.5$ years; 11 girls and 25 boys) and 19 with LD (mean= $6 \pm 1$ years; 7 girls and 12 boys). In order to avoid interpretation bias, we included only homogenous LD children with phonological-syntactic dysphasia and good oral language comprehension skills, based on Rapin and Allen classification of LD [19]. They were enrolled in medico-educational institutes, at-home intervention clinical services or in speech therapy private practices. Diagnoses were based on the DSM-5 criteria and were confirmed by clinicians in the Department of Child and Adolescent Psychopathology of the Pitié - Salpêtrière Hospital that have expertise in both ASD and LD [42].

\section{Material}

The computerised protocol was created by FB, a speech therapist, and implemented by a computer expert. The protocol consisted of thirty items divided into two categories: fifteen arbitrary gestures and fifteen iconic gestures (Table 1) that were defined according to the state of the art concerning typical children. The thirty gestures were realized by FB, without situational context, recorded on a video and introduced in the computerised protocol. These gestures were presented only visually i.e. without sound and word on a laptop computer in random order. The responses were presented visually (i.e. written) on the computer screen, read by the examiner and associated to colored tokens. The child could choose between three responses: the 'correct response', a 'literal response' and an 'incorrect response' corresponding to the same semantic field as the 'correct response' (Cf. Table 2). The presentation order of response modalities was randomised.

(PLEASE INSERT TABLE 1)

(PLEASE INSERT TABLE 2) 


\section{Procedure}

The testing of language gesture comprehension was performed on an individual basis. The child was alone in a quiet room with the examiner. He was placed in front of a laptop computer, and the examiner was sitting next to him. Videos with iconic or arbitrary gestures were presented to him. Each video sequence could be visualised several times, at the request of the child. Then, he was asked a question. For the iconic gestures, the child was asked, 'What is the person doing?' and, for the arbitrary gestures, 'What is the person saying?'. The child had to select the answer considered correct either by (i) clicking directly on the computer (ii) responding orally (iii) using the colored tokens. If the child answered spontaneously before the end of the response presentation, the examiner continued reading before selecting a final response to ensure consistency. When the child did not produce a response, the black box 'no response' was selected. For a wrong answer, the gesture was presented again and the software recorded the reaction time and responses.

\section{Statistical Analysis}

Statistical analyses were performed using R Software, Version 2.12.2. For every test, the level of significance, alpha, was fixed at 5\%. To assess the variables associated with the comprehension of conventional gestures in typical children, a binomial general linear mixed model (GLMM) was applied to the mean performance (two scores per subject). The three following variables were entered in the model: age (continuous variable); type of gesture (arbitrary vs. iconic); and gender (girl vs. boys). For each variable, the normal distribution was checked. To assess the performance of children with communication impairment, we used binomial general linear model (GLM) applied to the mean performance for arbitrary gesture then iconic gesture. The three following variables were entered in the models: age (continuous variable); type of group (TD vs. ASD vs. LD); and gender (girl vs. boys). Finally, we repeated the first analysis including individuals with communication impairments. To do so, we performed a binomial GLMM applied to the mean performance (two scores per subject). The four following variables were entered in the model: age (continuous variable); type of gesture (arbitrary vs. iconic); type of group (TD vs. ASD vs. LD) and gender (girl vs. boys). 


\section{RESULTS}

\section{Comprehension of Arbitrary and Iconic Gestures in Typical Children}

Figure 1 summarises the comprehension of $\mathrm{CG}$ for both arbitrary and iconic gestures in typical children according to age. The GLMM showed several significant effects: (i) comprehension significantly improved with age $(\beta=0.86, \mathrm{p}<.001)$; the secondary analyses on iconic gestures and arbitrary gestures separately showed that this was the case for both types of $\mathrm{CG}(\beta=0.042, \mathrm{p}<.001 ; \beta=0.043, \mathrm{p}<.001$, respectively) (ii) comprehension was significantly associated with the type of gesture: specifically, the comprehension of arbitrary gestures was significantly lower than that of iconic gestures $(\beta=0.44, \mathrm{p}<.001)$; the children were $1.6\left(=\mathrm{e}^{0.44}\right)$ times more likely to give a correct answer for iconic gestures vs. arbitrary ones; also, as shown in figure 1, the improvement of the comprehension of iconic gestures appeared earlier (nearly 6-month) than that of arbitrary ones (iii) comprehension tended to be significantly associated with gender, with boys showing better comprehension than girls: the secondary analyses on iconic gestures and arbitrary gestures separately showed that this was the case for iconic gestures only $(\beta=2.22, \mathrm{p}<.001)$, and not for arbitrary gestures $(\beta=0.07, \mathrm{p}=.28)$. The interaction between age and gender was significant $(\beta=0.04, \mathrm{p}<.001)$, indicating that, in the case of iconic gestures, improvement of comprehension with age was faster for girls (Figure 2).

\section{(PLEASE INSERT FIGURE 1) \\ (PLEASE INSERT FIGURE2)}

Comprehension of Arbitrary and Iconic Gestures in Children with Communication Impairments compared to TD children

Figure 3 summarises the comprehension of conventional gestures for both arbitrary and iconic gestures in typical children, children with ASD and children with LD For the iconic gestures separately, the GLM showed several significant effects: comprehension significantly improved with age $(\beta=0.042, \mathrm{p}<.001)$; comprehension was easier for girls $(\beta=0.33, \mathrm{p}<.001)$; comprehension was lower in children with ASD compared to TD Children $(\beta=-1.15$, $\mathrm{p}<.001)$; comprehension was higher in children with $\operatorname{LD}$ compared to TD children $(\beta=0.97, \mathrm{p}<.001)$; and comprehension was higher for LD compared to ASD children $(\beta=2.11, \mathrm{p}<.001)$. For the arbitrary gesture 
separately, the GLM showed several significant effects: comprehension significantly improved with age $(\beta=0.043, \mathrm{p}<.001)$; comprehension was lower in children with ASD compared to TD children $(\beta=-0.98, \mathrm{p}<.001)$; comprehension was higher in children with LD compared to TD children $(\beta=0.36, p=.023)$. However, there was no effect of gender $(\beta=0.023, p=.61)$ and comprehension was higher for LD compared to ASD children $(\beta=1.34$, $\mathrm{p}$ <.001). Finally, when we performed the binomial GLMM with both arbitrary and iconic gesture we found the same effects as for the model with typical children, only combined to the results found for children with communication impairments: (i) comprehension significantly improved with age $(\beta=0.87, \quad \mathrm{p}<.001)$; comprehension was lower in children with ASD compared to TD children $(\beta=-1.21, \mathrm{p}<.001)$; comprehension tended to be higher in children with LD compared to TD children $(\beta=0.51, \mathrm{p}=.058)$ but was significantly higher when compared to ASD $(\beta=1.72, \mathrm{p}<.001)$; comprehension was easier for iconic gestures compared to arbitrary ones $(\beta=0.42, p<.001)$. However, there was no effect of gender $(\beta=-0.17, p=.15)$.

(PLEASE INSERT FIGURE 3) 


\section{DISCUSSION}

The first aim of the present study was to analyse the developmental course of conventional gestures (CG) comprehension in typical children and in children with communication impairments (ASD and LD). As expected, conventional gesture comprehension increased during the early development of typical children, and the improvement of iconic gestures comprehension occurred earlier than that of arbitrary ones. The second aim of the study was, in a clinical perspective, to document how children with language impairments and developmental communication disorders can understand $\mathrm{CG}$, to the extent that this ability can compensate for language weakness or absence. Children with ASD received lower CG scores than typical children and LD children. But, contrary to our fourth hypothesis, for each gesture taken separately, children with LD performed better than, not only ASD children, but typical children as well. Furthermore, when we performed the analysis with both arbitrary and iconic gesture, children with LD outperformed both children with ASD and TD children.

\section{Developmental Course of Conventional Gesture Comprehension}

In TD children from age 3 to 10 years, for both arbitrary and iconic gestures, gesture comprehension significantly increased during the children's development. This age effect is explained by the fact that the development of conjoint activity throughout daily interactions allows children to socialise with communicative intentions and, thus, progressively adapt the acquired codes to various social situations. These interaction patterns represent a fundamental resource to transfer from non-verbal communication to oral language. With the emergence and use of oral language, the child's gesture repertoire is transformed and enriched similarly to the lexical, semantic and pragmatic development of language [43]. Due to acquired experiences during childhood, several CG subsist in older children and adults because they represent a common code [14]. In addition, we found that comprehension of conventional gestures was influenced by gesture type and gender.

Concerning gesture type, in line with Dimitrova et al. (2017), comprehension was better for iconic than for arbitrary gesture. This result is consistent with the cognitive development of children in view of the concrete nature of iconic gestures [16]. The iconic gestures were easier to understand because they represent and directly suggest children's daily activities. Because children are confronted daily with iconic gestures at an early age, they quickly memorise the constant relationship between significant and signified, which facilitates comprehension. This situation is also the case for several arbitrary gestures ('congratulations', 'be quiet', and 'goodbye') that are often practiced during daily interactions and, thus, can be quickly memorised. In contrast, most arbitrary gestures are less frequently used in daily interactions and are less concrete, depending on the 
progress of memory capacity and the quality and intensity of child-adult interactions. Finally, our results are consistent with those of Namy [17] showing that, contrary to iconic gestures, arbitrary gestures are not as well understood between 3 and 4 years old.

Concerning gender, our data showed a gender effect limited to iconic gestures. This result contrasts with Botting's study [27] which found no gender differences in gesture scores, but gender effect has been very seldom studied in a developmental way in the literature. We found that boys displayed better comprehension than girls who showed a faster improvement of comprehension with age (Figure 2). This could be due to the fact that girls tend to develop language earlier and better than boys [44] and, thus, tend to use fewer gestures to communicate. Boys compensate a slower maturation of oral language with a higher level of motor and gesture activity. In addition, parents play a key role in motor skills development by placing more importance on the physical development of boys [45]. Moreover, the sex of the parent and the playful context (e.g., use of certain toys) seem to have an impact on the language development in favour of girls [46]. Thus, the variables concerning biology and social and developmental contexts could contribute to this gender effect [47].

\section{Conventional Gesture Comprehension in Children with LD}

All statistical analyses (whether they explored both arbitrary and iconic gestures or each gesture separately), reported that comprehension was higher for LD compared to both TD and ASD. The comparison with ASD is an expected result knowing the severe deficits in communication shown by children with ASD. The comparison with TD contradicts the theory of an integrated communication system [4] between gesture and speech that argue difficulties with gestural communication in children with LD. In this study, children with LD had rather good comprehension skills and mainly phonological-syntactic deficits. They appeared to rely on conventional gestures to understand and interpret the communicative intentions of others. All of these gestures that replace words and allow children to express information constitute a mechanism that compensates for language deficits $[24,27]$. When children with LD must process simultaneously speech and gestures, they may have difficulties because of cross-modal processing limitations $[26,27]$ and, thus, they often prefer to interpret gestures. Moreover, children with LD more easily produce gestures than words when the context allows them to do so [24]. At an early age, children with LD use symbolical gestures similar to the typical children; however, when growing up, they continue to depend on information produced by gestures more than by words because of their language delay. Similarly, children with LD produce more iconic gestures than the typical children [20,23]. The interpretation of conventional gestures allows them to facilitate social interactions that are disturbed but not hindered by their 
language impairment. To conclude, discrepancies in earlier studies regarding CG in LD (see introduction) may be related to language domain deficit (e.g. lexicon) or language comprehension deficit of children with LD included in these reports. Therefore, generalisation of our results needs the study to be replicated in a larger sample.

\section{Conventional Gesture Comprehension in Children with ASD}

Children with ASD performed worse in conventional gesture comprehension than typical children of similar developmental age. The association between action and its representation with iconic gestures did not facilitate comprehension in this group. It appeared that those children were unable to integrate the symbolic sense of conventional gestures and their meaning because they experienced difficulty using them outside social routines and generalising their use out of a particular context [48]. In addition to communication abilities, the comprehension and use of CG require imitation skills. Imitation skills play a fundamental role in the development of communication and may constitute an initial social signature during interaction [49]. Indeed, as long as children have no access to oral language, they use imitation to communicate [50] and to learn using conventional gestures. Tomasello and Camaioni hypothesised that children with ASD have imitation difficulties, which impairs conventional gesture acquisition [51]. This imitation deficit could be explained by a weak interest in social interaction and the presence of severe executive impairments, more than by a deficit per se of imitation [50]. Children with ASD also show heterogeneous performance in facial emotion processing [52,53] that play a role in the comprehension of verbal and non-verbal messages. All of the communicative and emotional difficulties observed in children with ASD could be underlined by two factors. First, according to the 'weak central coherence theory' which was conceptualized by Happé and Frith [54] and corresponds to the difficulties that children with ASD have when synthesizing stimuli into a coherent whole, children with ASD have impairments in multisensory processing [55]. Indeed, patients with ASD would not globally perceive the stimuli and, consequently, they would not establish the coherence of the object they observe. Information processing would be local and unorganised. Second, a deficit in TOM (theory of mind) could explain some aspects of communication disorders [56]. Children with ASD have difficulties deducing the mental states of others, including their thoughts, intentions and emotions. They are occasionally capable of performing this function, however they do so later and less efficiently compare with typical children.

\section{Study Limitations and Strengths}


The results of the current study should be interpreted according to certain limitations. First, in contrast with the large sample of typical children, the group of atypical children was restricted. Specifically, the sub-analyses according to age had a restricted number of pathological cases, which limits the interpretation of clinical findings. Second, because the inclusion criteria precluded the existence of pointing gestures and a level of comprehension superior to children aged 3 years to ensure participation in the protocol, many young or severely pathological children were excluded from the study. In our procedure the gestures integrated different levels of complexity (e.g., sleeping gesture much easier than playing flute) that we did not take into account in our results. According to the idea that gestures are a complex skill,in ecological situation as well as in our experimental setup, the majority of gestures accompany facial expressions, which give cues for interpreting and comprehending conventional gesture. For instance, in the current protocol, the experimenter's face expressed anger (e.g. 'things are going badly') or happiness (e.g. 'congratulations'). Facial expressions that were used to understand the psychological state of the interlocutor optimised the social interaction regulation. They sustained behaviour adjustment and facilitated communication between the child and others [57]. It can be hypothesised that children's comprehension of gestures benefits from these facial cues. Besides, although the children had similar school level, there were no systematic measures of the IQ, precluding controlling for IQ. In addition, although the team is specialised in the field of autism, the fact that the diagnoses of autism spectrum disorder were not established by standardised evaluation but only through clinical evolution can constitute a limitation.

On the other hand, this study presents several interesting preliminary findings. It shows the overall validity of the computer-based protocol from a clinical perspective. This study is now being finalised using a new protocol that combines conventional gesture comprehension and visual processing (eye tracking). The aim is to study (i) how children visually explore the videos to access conventional gesture comprehension (ii) whether the synchrony between correct responses and gaze direction in typical children and children with communication disorders (ASD or LD) may allow us to passively assess CG comprehension in young children with no language and no pointing gestures.

\section{Conclusion}

Among typical children, $\mathrm{CG}$ comprehension improves from 3 to 10 years old. The improvement of the CG comprehension occurs earlier for iconic gestures compared to arbitrary gestures. Among children with developmental communication impairments, two developmental profiles can be distinguished. First, children with ASD have lower performance compared to typical children. In contrast, children with LD perform better 
than both typical children and children with ASD. These results suggest a dissociation between oral language comprehension and language gesture comprehension in children with LD and a deficit in both oral language and language gesture comprehension in children with ASD.

\section{Acknowledgments}

The study was supported by the Agence Nationale de la Recherche (ANR-12-SAMA-006), the Centre

d'Activités en Psychiatrie Infanto-Juvenile and the Groupement de Recherche en Psychiatrie (GDR-3557).

Sponsors had no involvement in the study design, data analysis, or interpretation of the results.

\section{Conflict of interest}

The authors declare that the research was conducted in the absence of any commercial or financial relationships that could be construed as a potential conflict of interest. 


\section{References}

1. Bernicot, J. Introduction. L’usage Gestes Mots Chez L'enfant. Colin. Paris; 1998. p. 5-25.

2. Capirci O, Iverson JM, Pizzuto E, Volterra V. Gestures and words during the transition to two-word speech. J. Child Lang. 1996;23:645-73.

3. Goldin-Meadow S, Alibali MW. Gesture's role in speaking, learning, and creating language. Annu. Rev. Psychol. 2013;64:257-83.

4. Mc Neill D. Hand and mind: what gestures reveal about thought [Internet]. Chicago: University of Chicago Press; $1992 \quad$ [cited $2017 \quad$ May 13$]. \quad$ Available from: http://www.cogsci.ucsd.edu/ nunez/COGS155_W17/McNeill_CH3_PS.pdf

5. Kendon A. Gesture: Visible Action as Utterance. Cambridge University Press; 2004.

6. Guidetti M. Yes or no? How young French children combine gestures and speech to agree and refuse. J. Child Lang. 2005;32:911-24.

7. Ekman P., Friesen W. The repertoire of Non Verbal Behaviour, Categories, Origins, Usage and Coding [Internet]. $1969 \quad$ [cited $2017 \quad$ May 13$]. \quad$ Available from: http://homes.dsi.unimi.it/ boccignone/GiuseppeBoccignone_webpage/CompAff2011_files/EkmanFriesenSemiot ica.pdf

8. Özçalıkan E, Goldin-Meadow S. Gesture is at the Cutting Edge of Early Language Development. Cognition. 2005;96:101-13.

9. Iverson JM, Goldin-Meadow S. Gesture paves the way for language development. Psychol. Sci. 2005;16:36771.

10. Bates E. The Emergence of Symbols: Cognition and Communication in Infancy. Academic Press; 1979.

11. Ekman P. Movements with Precise Meanings. J. Commun. 1976;26:14-26.

12. Goodwyn SW, Acredolo LP, Brown CA. Impact of Symbolic Gesturing on Early Language Development. J. Nonverbal Behav. 2000;24:81-103.

13. Kumin L, Lazar M. Gestural Communication in Preschool Children. Percept. Mot. Skills. 1974;38:708-10.

14. Guidetti M. Aspects pragmatiques de la communication gestuelle et verbale chez le jeune enfant: une comparaison France/Côte-d'Ivoire, Pragmatic aspects of gestural and verbal communication in young children: A comparison France/Ivory Coast. Enfance. 2006;58:169-77.

15. Guidetti M. The emergence of pragmatics: forms and functions of conventional gestures in young French children. First Lang. 2002;22:265-85.

16. Piaget J. Play, dreams and imitation in childhood. [Internet]. 1952 [cited 2017 May 13]. Available from: http://web.media.mit.edu/ ascii/papers/piaget_1952.pdf

17. Namy L.L, Campbell A.L., Tomasello M. The Changing Role of Iconicity in Non-Verbal Symbol Learning : A U-Shaped Trajectory in the Acquisition of Arbitrary Gestures. J. Cogn. Dev. [Internet]. 2004; Available from: https://www.researchgate.net/publication/27283513_The_Changing_Role_of_Iconicity_in_NonVerbal_Symbol_Learning_A_U-Shaped_Trajectory_in_the_Acquisition_of_Arbitrary_Gestures

18. Bishop DV, Chan J, Adams C, Hartley J, Weir F. Conversational responsiveness in specific language impairment: evidence of disproportionate pragmatic difficulties in a subset of children. Dev. Psychopathol. 2000;12:177-99.

19. Rapin I., Allen D.A. Developmental language: nosological consideration. Elsevier; 1983. 
20. Iverson JM, Braddock BA. Gesture and motor skill in relation to language in children with language impairment. J. Speech Lang. Hear. Res. JSLHR. 2011;54:72-86.

21. Stothard SE, Snowling MJ, Bishop DV, Chipchase BB, Kaplan CA. Language-impaired preschoolers: a follow-up into adolescence. J. Speech Lang. Hear. Res. JSLHR. 1998;41:407-18.

22. Powell RP, Bishop DV. Clumsiness and perceptual problems in children with specific language impairment. Dev. Med. Child Neurol. 1992;34:755-65.

23. Mainela-Arnold E, Alibali MW, Hostetter AB, Evans JL. Gesture-speech integration in children with specific language impairment. Int. J. Lang. Commun. Disord. R. Coll. Speech Lang. Ther. 2014;49:761-70.

24. Blake J, Myszczyszyn D, Jokel A, Bebiroglu N. Gestures accompanying speech in specifically languageimpaired children and their timing with speech. First Lang. 2008;28:237-53.

25. Hart KI, Fujiki M, Brinton B, Hart CH. The relationship between social behavior and severity of language impairment. J. Speech Lang. Hear. Res. JSLHR. 2004;47:647-62.

26. Wray C, Norbury CF, Alcock K. Gestural abilities of children with specific language impairment. Int. J. Lang. Commun. Disord. 2016;51:174-82.

27. Botting N, Riches N, Gaynor M, Morgan G. Gesture production and comprehension in children with specific language impairment. Br. J. Dev. Psychol. 2010;28:51-69.

28. Saint-Georges C, Guinchat V, Chamak B, Apicella F, Muratori F, Cohen D. Signes précoces d'autisme: D'où vient-on? Où va-t-on? Neuropsychiatr. Enfance Adolesc. 2013;61:400-8.

29. Paul R. Language Disorders from Infancy Through Adolescence: Assessment \& Intervention. Elsevier Health Sciences; 2007.

30. Wing L. Asperger's syndrome: a clinical account. Psychol. Med. 1981;11:115-29.

31. Özçalışkan Ş, Adamson LB, Dimitrova N. Early deictic but not other gestures predict later vocabulary in both typical development and autism. Autism Int. J. Res. Pract. 2016;20:754-63.

32. Charman T, Drew A, Baird C, Baird G. Measuring early language development in preschool children with autism spectrum disorder using the MacArthur Communicative Development Inventory (Infant Form). J. Child Lang. 2003;30:213-36.

33. Luyster R, Lopez K, Lord C. Characterizing communicative development in children referred for autism spectrum disorders using the MacArthur-Bates Communicative Development Inventory (CDI). J. Child Lang. 2007;34:623-54.

34. Bartak L, Rutter M, Cox A. A comparative study of infantile autism and specific development receptive language disorder. I. The children. Br. J. Psychiatry J. Ment. Sci. 1975;126:127-45.

35. Colgan SE, Lanter E, McComish C, Watson LR, Crais ER, Baranek GT. Analysis of social interaction gestures in infants with autism. Child Neuropsychol. J. Norm. Abnorm. Dev. Child. Adolesc. 2006;12:307-19.

36. Lord C, Risi S, Lambrecht L, Cook EH, Leventhal BL, DiLavore PC, et al. The autism diagnostic observation schedule-generic: a standard measure of social and communication deficits associated with the spectrum of autism. J. Autism Dev. Disord. 2000;30:205-23.

37. Lord C, Rutter M, Le Couteur A. Autism Diagnostic Interview-Revised: a revised version of a diagnostic interview for caregivers of individuals with possible pervasive developmental disorders. J. Autism Dev. Disord. 1994;24:659-85.

38. Robins DL, Fein D, Barton ML, Green JA. The Modified Checklist for Autism in Toddlers: an initial study investigating the early detection of autism and pervasive developmental disorders. J. Autism Dev. Disord. 2001;31:131-44. 
39. Mitchell S, Brian J, Zwaigenbaum L, Roberts W, Szatmari P, Smith I, et al. Early language and communication development of infants later diagnosed with autism spectrum disorder. J. Dev. Behav. Pediatr. JDBP. 2006;27:S69-78.

40. Camaioni L, Perucchini P, Muratori F, Parrini B, Cesari A. The communicative use of pointing in autism: developmental profile and factors related to change. Eur. Psychiatry. 2003;18:6-12.

41. LeBarton ES, Iverson JM. Gesture development in toddlers with an older sibling with autism. Int. J. Lang. Commun. Disord. 2016;51:18-30.

42. Demouy J, Plaza M, Xavier J, Ringeval F, Chetouani M, Périsse D, et al. Differential language markers of pathology in Autism, Pervasive Developmental Disorder Not Otherwise Specified and Specific Language Impairment. Res. Autism Spectr. Disord. 2011;5:1402-12.

43. Colletta J.M., Pellenq C., Guidetti M. Age-related changes in co-speech gesture and narrative: Evidence from French children and adults. Speech Commuication. 2010;52(6):565-76.

44. Bouchard C., Trudeau N., Sutton A., Boudreault M.C., Deneault J. Gender differences in language development in French Canadian children between 8 and 30 months of age. Appl. Psycholinguist. 2009;685707.

45. Eliot L. Cerveau rose, cerveau bleu: les neurones ont-ils uns sexe? Robert Laffont. Paris; 2011.

46. Bailly, S. «Les filles sont plus douées pour les langues? »: enquête autour d'une idée reçue. Mélanges CRAPEL. 1993.

47. Leaper C, Smith TE. A meta-analytic review of gender variations in children's language use: talkativeness, affiliative speech, and assertive speech. Dev. Psychol. 2004;40:993-1027.

48. Bernabei P, Camaioni L. Developmental profile and regression in a child with autism: a single case study. Autism Int. J. Res. Pract. 2001;5:287-97.

49. Boucenna S., Anzalone S., Tilmont T., Cohen D., Chetouani M., the Michelangelo Study Group. Extraction of social signatures through imitation learning between a robot and a human partner. IEEE Trans. Auton. Ment. Dev. 2014;6 (3):213-25.

50. Nadel J. How imitation boosts development in infancy and Autism Spectrum Disorder [Internet]. Oxford: Oxford University Press; 2014 [cited 2017 May 14]. Available from: https://www.researchgate.net/publication/265122846_Nadel_J_2014_How_imitation_boosts_development_in_in fancy_and_Autism_Spectrum_Disorder_Oxford_Oxford_University_Press

51. Tomasello M, Camaioni L. A Comparison of the Gestural Communication of Apes and Human Infants. Hum. Dev. 1997;40:7-24.

52. Vannetzel L, Chaby L, Cautru F, Cohen D, Plaza M. Neutral versus emotional human stimuli processing in children with pervasive developmental disorders not otherwise specified. Res. Autism Spectr. Disord. 2011;5:775-83.

53. Xavier J, Vignaud V, Ruggiero R, Bodeau N, Cohen D, Chaby L. A Multidimensional Approach to the Study of Emotion Recognition in Autism Spectrum Disorders. Front. Psychol. [Internet]. 2015 [cited 2017 May 14];6. Available from: http://www.ncbi.nlm.nih.gov/pmc/articles/PMC4689801/

54. Happé F, Frith U. The weak coherence account: detail-focused cognitive style in autism spectrum disorders. J. Autism Dev. Disord. 2006;36:5-25.

55. Collignon O, Charbonneau G, Peters F, Nassim M, Lassonde M, Lepore F, et al. Reduced multisensory facilitation in persons with autism. Cortex J. Devoted Study Nerv. Syst. Behav. 2013;49:1704-10.

56. Baron-Cohen S, Leslie AM, Frith U. Does the autistic child have a "theory of mind"? Cognition. $1985 ; 21: 37-46$ 
57. Ekman P., Friesen W., Ellsworth P. Conceptual ambiguities. In : Emotion in Human Face. Editions de la Maison des Sciences de 1'Homme. Paris; 1982. 


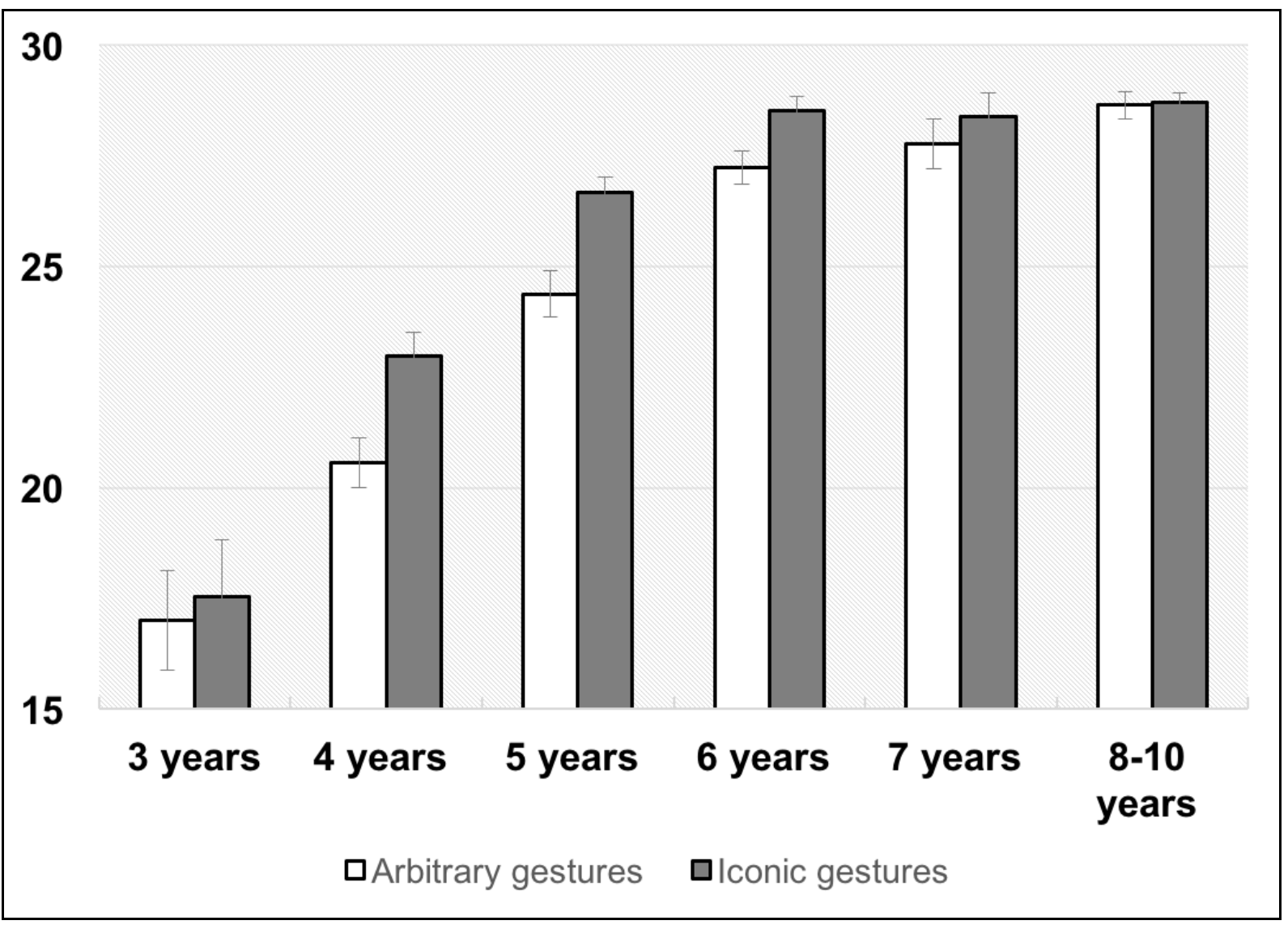

FIGURE 1. Mean number of correct answers for arbitrary and iconic gestures in typical children 


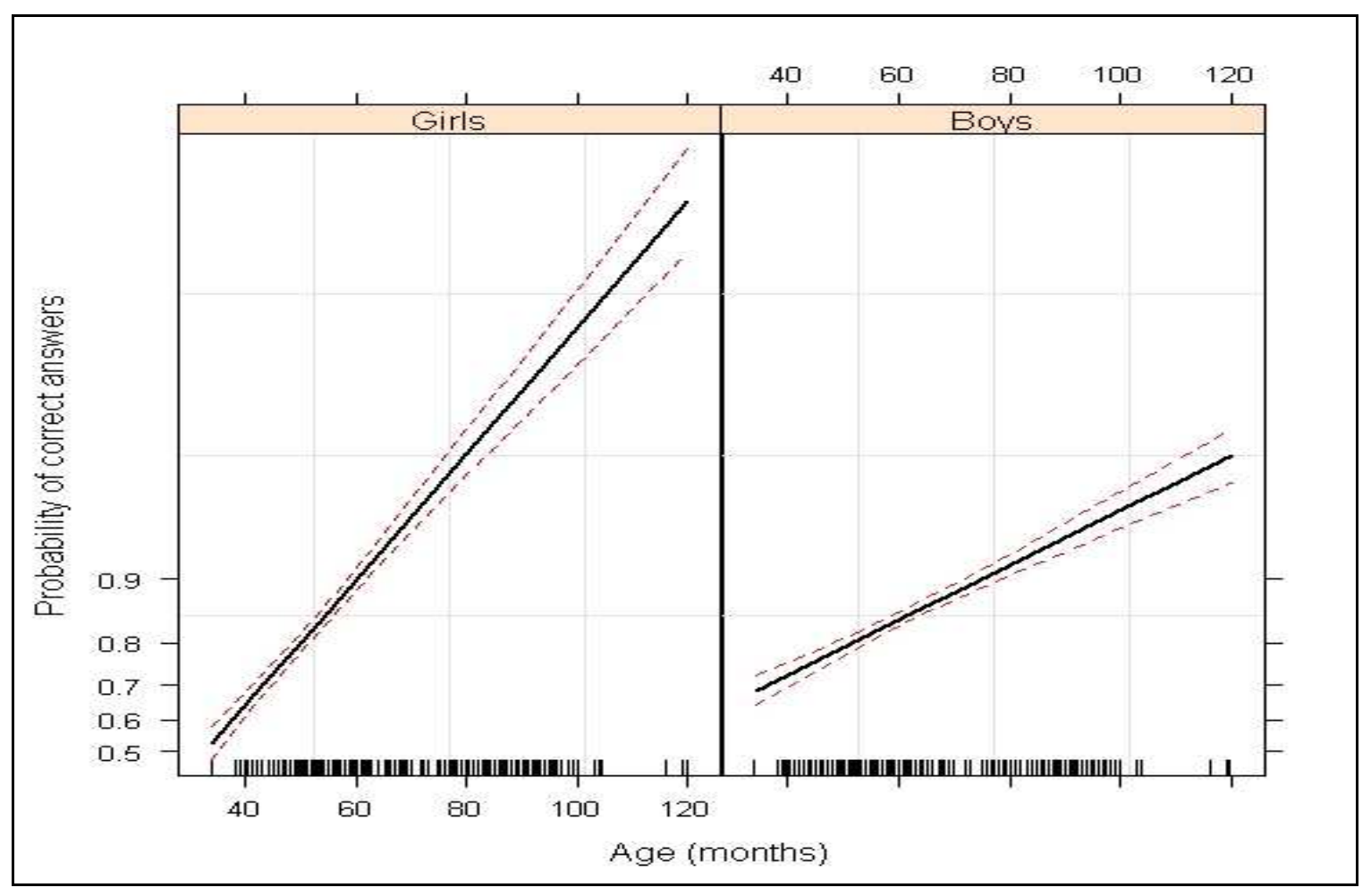

FIGURE 2. Probability of correct answers for iconic gestures in typical girls and boys 


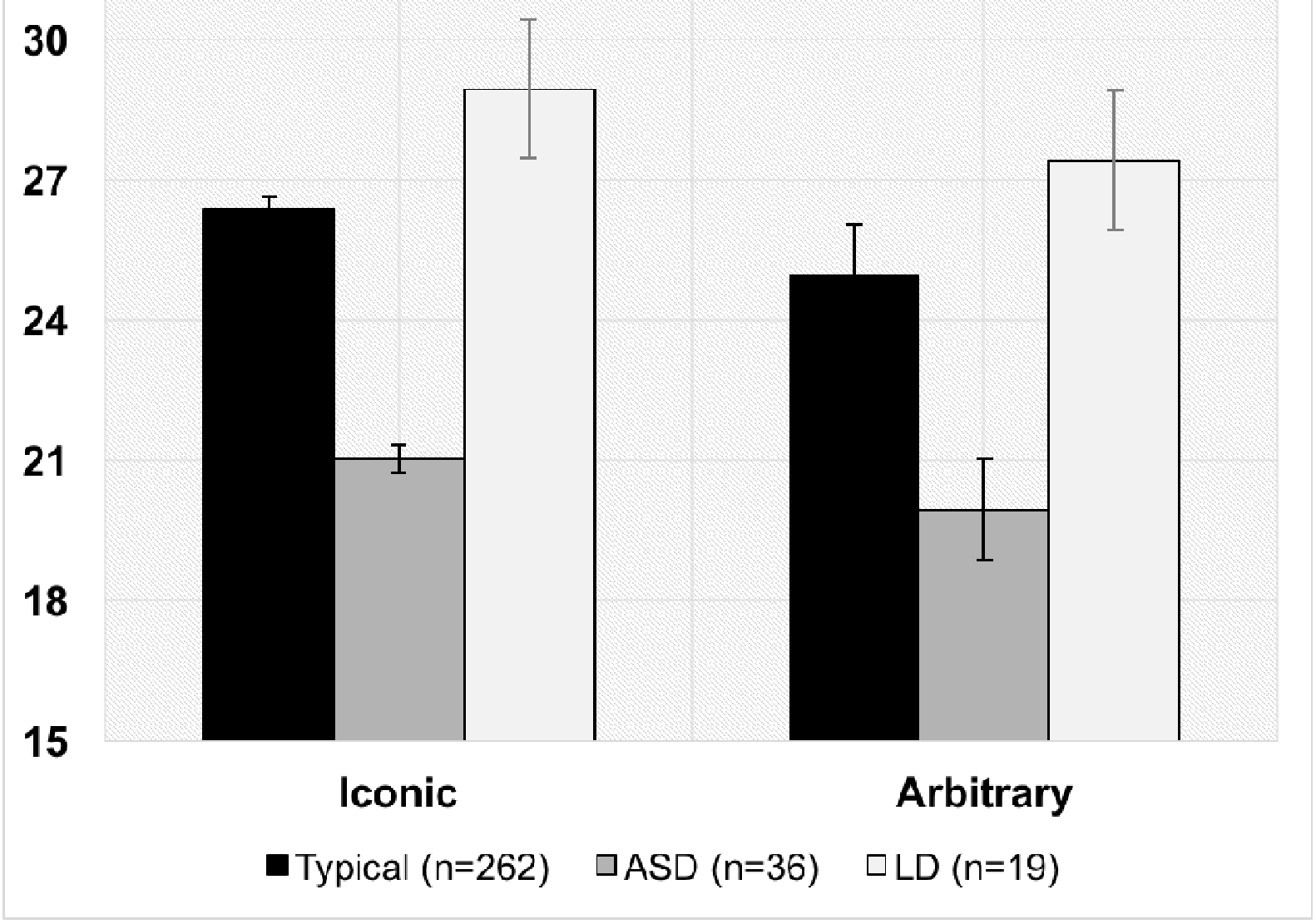

FIGURE 3. Mean number of correct answers for arbitrary and iconic gestures in typical children and children with $A S D$ and $L D$ 
TABLE 1. List of thirty conventional gestures (15 iconic and 15 arbitrary gestures)

\begin{tabular}{|l|l|}
\hline \multicolumn{1}{|c|}{ Iconic gestures } & \multicolumn{1}{c|}{ Arbitrary gestures } \\
\hline She drinks & Goodbye \\
She eats & I don't know \\
She sleeps & You're crazy \\
She cuts & Go away \\
She calls & I have enough \\
She drives & I love you \\
She ties her shoe laces & Ouch \\
She writes & Yes \\
She combs her hair & No \\
She plays piano & Come \\
She brushes her teeth & Great \\
She washes her hands & Quiet \\
She reads & Stop \\
She paints & Congratulations \\
She plays flute & Things are going badly \\
\hline
\end{tabular}


TABLE 2. Item and response examples for iconic and arbitrary gestures

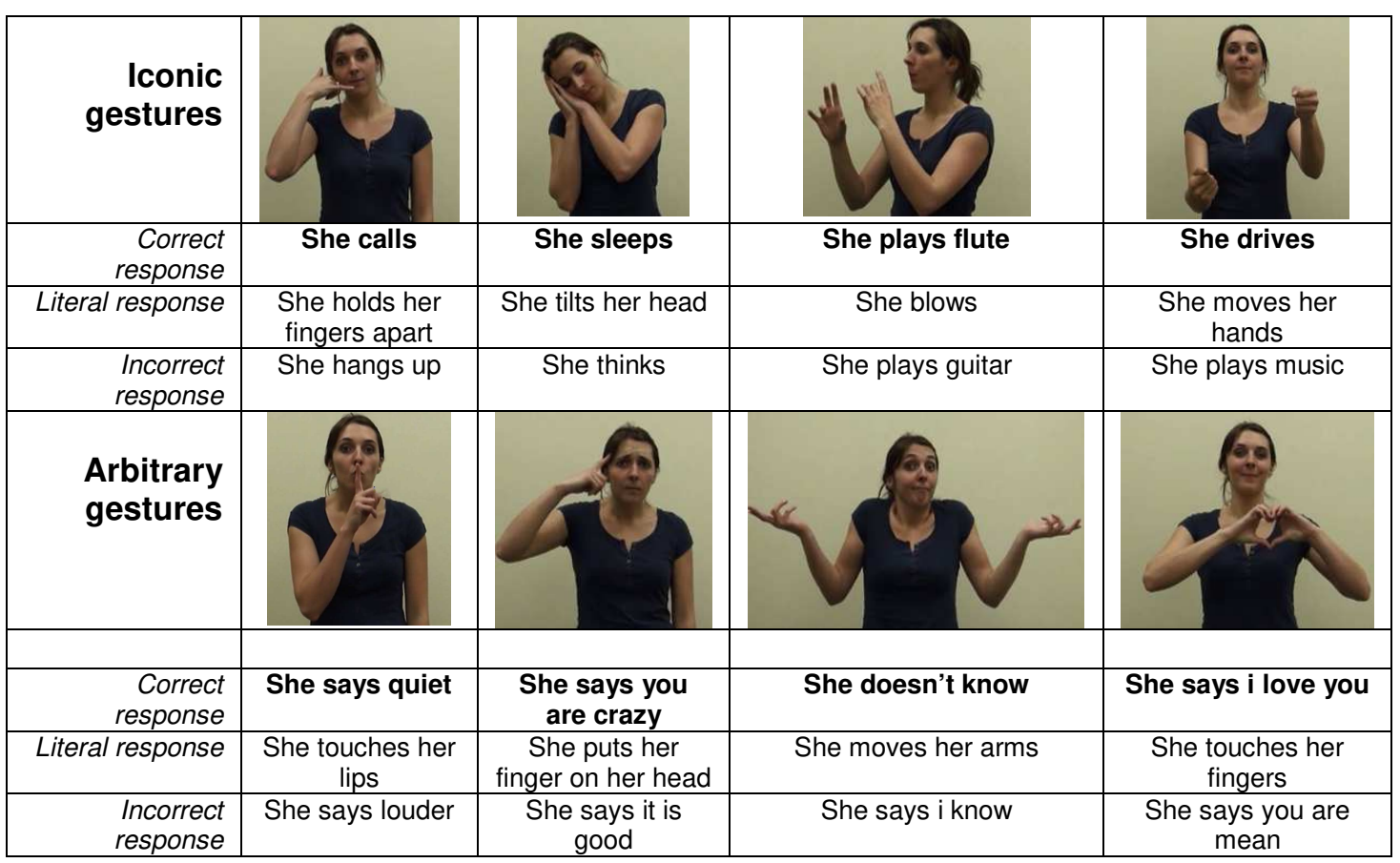

